\title{
Thalamocingulate Interactions In Performance Monitoring
}

\author{
Sebastian Seifert, ${ }^{1}$ D. Yves von Cramon, ${ }^{1,2}$ Davide Imperati, ${ }^{2}$ Marc Tittgemeyer, ${ }^{2}$ and Markus Ullsperger ${ }^{2,3}$ \\ ${ }^{1}$ Max Planck Institute for Human Cognitive and Brain Sciences, 04303 Leipzig, Germany, ${ }^{2}$ Max Planck Institute for Neurological Research, 50931 Cologne, \\ Germany, ${ }^{3}$ Radboud University Nijmegen, Donders Institute for Brain, Cognition and Behaviour, 6500 Nijmegen, The Netherlands
}

Performance monitoring is an essential prerequisite of successful goal-directed behavior. Research of the last two decades implicates the anterior midcingulate cortex (aMCC) in the human medial frontal cortex and frontostriatal basal ganglia circuits in this function. Here, we addressed the function of the thalamus in detecting errors and adjusting behavior accordingly. Using diffusion-based tractography, we found that, among the thalamic nuclei, the ventral anterior (VA) and ventral lateral anterior (VLa) nuclei have the relatively strongest connectivity with the aMCC. Patients with focal thalamic lesions showed diminished error-related negativity, behavioral error detection, and posterror adjustments. When the lesions specifically affected the thalamic VA/VLa nuclei, these effects were significantly pronounced, which was reflected by the complete absence of the error-related negativity. These results reveal that the thalamus, particularly its VA/VLa region, is a necessary constituent of the performance-monitoring network, anatomically well connected and functionally closely interacting with the aMCC.

\section{Introduction}

Human goal-directed behavior requires a flexible system evaluating behavioral progress and adjusting behavior as needed. The error-related negativity (ERN), an event-related brain potential (ERP) associated with response errors (Falkenstein et al., 1990; Gehring et al., 1993), is used to study the function of this performance-monitoring system (Ullsperger, 2006). ERN is assumed to be generated in the anterior midcingulate cortex (aMCC) (Debener et al., 2005) and to reflect a discrepancy or conflict of erroneous and competing correct response tendencies (Falkenstein et al., 1990; Coles et al., 2001; Yeung et al., 2004). The aMCC overlaps with the rostral cingulate zone (Picard and Strick, 1996), the human homolog of the monkey's rostral and dorsal cingulate motor areas. Neuroimaging and invasive studies in humans and monkeys showed that the aMCC is part of a larger network signaling the need for behavioral change (Shima and Tanji, 1998; Ridderinkhof et al., 2004), thereby taking into account outcome history (Kennerley et al., 2006; Rushworth and Behrens, 2008; Jocham et al., 2009). Studies in patients with focal lesions (Gehring and Knight, 2000; Ullsperger et al., 2002; Hogan et al., 2006; Ullsperger and von Cramon, 2006a), Parkinson's disease (Falkenstein et al., 2001; Frank et al., 2004, 2007; Willemssen et al., 2008), and Huntington's disease (Beste et al., 2006, 2008) provide strong evidence that interactions of the aMCC with the lateral prefrontal cortex and the basal ganglia (BG) are necessary for functional integrity of performance monitoring. Presumably, the BG play a key role in monitoring and adjusting performance-ranging from immediate motor adjustments via

Received Nov. 30, 2010; revised Jan. 2, 2011; accepted Jan. 10, 2011.

This work was supported by a grant from the Deutsche Forschungsgemeinschaft (KF0 219, UL196/2-1, to M.U.). Correspondence should be addressed to Prof. Dr. Markus Ullsperger, Donders Institute for Brain, Cognition and Behaviour, Centre for Cognition, Radboud University Nijmegen, P.O. Box 9104, 6500 Nijmegen, The Netherlands. E-mail: m.ullsperger@donders.ru.nl.

DOI:10.1523/JNEUROSCI.6242-10.2011

Copyright $\odot 2011$ the authors $\quad 0270-6474 / 11 / 313375-09 \$ 15.00 / 0$ action selection to learning from action outcomes (Holroyd and Coles, 2002; Frank, 2005). In the BG circuits relevant for performance monitoring, the thalamus seems to enable information flow from the prefrontal cortex to the more motor-related areas via nonreciprocal corticothalamic connections (Haber, 2003). Moreover, the ventral anterior (VA) and ventrolateral anterior (VLa) thalamus appears to mediate the interaction of cerebellar inputs with the BG loops to "adaptively adjust basal ganglia activity on the basis of some internal model and error signal" (Hoshi et al., 2005). Here, we investigate the role of the thalamus in human performance monitoring by studying its anatomical connectivity with the aMCC and examining functional impairments related to focal thalamic lesions.

Evidence from nonhuman primates suggests that the aMCC receives inputs from the adjacent pre-supplementary motor area, dorsal premotor cortex, and the thalamic VA and VLa nuclei (Van Hoesen et al., 1993; Hatanaka et al., 2003). These nuclei, in turn, are termination sites of pallidal efferents (Dum and Strick, 1993). Thus, the aMCC receives input from BG predominantly via the thalamic VA/VLa region. In addition, mediodorsal (MD), anterior, and intralaminar nuclei connect to aMCC (Vogt et al., 1979; Baleydier and Mauguiere, 1980; Barbas et al., 1991). Using diffusion-based tractography, we tested whether fiber connections between aMCC and thalamus originate in the VA/VLa region in humans. In addition, the functional role of the thalamus and its subregions to performance monitoring were tested in an ERP study with patients suffering from focal thalamic lesions.

\section{Materials and Methods}

\section{Diffusion-based imaging}

Participants. Sixteen healthy participants (five female; mean age, 29.6 years; age range, 25-38 years) took part in the diffusion data acquisition, which was performed at the Max-Planck-Institute for Human Cognitive and Brain Sciences in Leipzig, Germany. Subjects had no history of neurological, psychiatric, or other major medical disorder. The experiments were conducted in accordance with the Declaration of Helsinki and the study was approved by the local ethics committee of the University of 
Table 1. Detailed demographic and lesion data of the investigated patients

\begin{tabular}{|c|c|c|c|c|c|c|c|c|}
\hline Patient ID & Sex & Age (years) & Time since lesion (years) & Lesion side & Etiology & Lesion description & VA/VLa & MD \\
\hline \multicolumn{9}{|c|}{ VA/VLa group } \\
\hline P011 & M & 53 & 9 & $\mathrm{~L}$ & MCAI & ventr.-lat. Th & + & - \\
\hline P325 & M & 45 & 7 & B & MCAl, after AVM & ventr.-lat. Th (B), LPFC (L) & + & + \\
\hline P438 & M & 54 & 5 & $\mathrm{~L}$ & MCAI & ventr.-lat. Th & + & - \\
\hline P925 & M & 27 & 4 & L & ICH, cavernoma & ventr.-lat. Th, ant. Put, GPe, IC & + & - \\
\hline P1082 & $\mathrm{F}$ & 45 & 2,5 & $\mathrm{R}$ & ACl occl. & ventr.-lat. Th, Cd, GPe, IC (knee) & + & - \\
\hline P1145 & M & 56 & 1 & L & $\mathrm{ICH}$ & lat. Th, IC & + & - \\
\hline \multicolumn{9}{|c|}{ Non-VA/VLa group } \\
\hline P207 & M & 65 & 8 & L & MCAI & med. Th & - & + \\
\hline P214 & M & 54 & 6 & $\mathrm{~L}$ & ICH & post.-lat. Th, post. Put, GP, IC & - & + \\
\hline P575 & M & 41 & 2,5 & B & MCAI & med. Th & - & + \\
\hline P789 & $\mathrm{F}$ & 23 & 3 & $\mathrm{R}$ & MCAI & post. Th, post. Put, Cd, IC & - & - \\
\hline P845 & M & 41 & 2,5 & $\mathrm{R}$ & MCAI & ventr.-lat.-med. Th & - & + \\
\hline P1004 & $\mathrm{F}$ & 56 & 1 & L & MCAI & med. Th & - & + \\
\hline P1168 & $\mathrm{F}$ & 19 & 5 & L & TE, pinealoma & post. Th & - & - \\
\hline P1263 & $F$ & 44 & 0,5 & L & $\mathrm{ICH}$ & post. Th & - & + \\
\hline P1298 & M & 48 & 2 & R & LI & med. Th & - & + \\
\hline
\end{tabular}

M, Male; F, female; L, left; R, right; B, bilateral; MCAl, middle cerebral artery infarction; AVM, arteriovenous malformation; ICH, intracerebral hemorrhage; LI, lacunar infarctions; TE, tumor excision; ventr., ventral; lat., lateral; ant., anterior; med., medial; post., posterior; Th, thalamus; LPFC, lateral prefrontal cortex; Put, putamen; IC, internal capsule; Ge, globus pallidus; GPe, globus pallidus externus; Cd, caudate nucleus; + , lesion present; --, no lesion.

Leipzig (Leipzig, Germany). Participants gave written informed consent. Data were handled anonymously.

Data acquisition and preprocessing. Diffusion-weighted data and highresolution three-dimensional (3D) T1- and T2-weighted images were acquired on a Siemens 3T Trio scanner with an eight-channel array head coil and maximum gradient strength of $40 \mathrm{mT} / \mathrm{m}$. The diffusionweighted data were acquired using twice-refocused spin-echo echo planar imaging (repetition time $=12 \mathrm{~s}$, echo time $=100 \mathrm{~ms}, 72$ axial slices, resolution $1.72 \times 1.72 \times 1.7 \mathrm{~mm}$ ). We used a GRAPPA technique (reduction factor 2.0) for parallel imaging. Diffusion weighting was isotropically distributed along 60 directions (Jones et al., 1999) with a $b$ value of $1000 \mathrm{~s} / \mathrm{mm}^{2}$. The high angular resolution of the diffusion-weighting directions improves the robustness of probability density estimation by increasing the signal-to-noise ratio and reducing directional bias. Additionally, seven datasets with no diffusion weighting (b0) were acquired initially and interleaved after each block of 10 diffusion-weighted images as anatomical reference for motion correction. To further increase signal-to-noise, we acquired three consecutive scans, which were subsequently averaged together. The entire data acquisition protocol lasted $\sim 45$ min. Motion correction for the diffusion-weighted images was applied to all images using seven-parameter global rescale registration (Jenkinson et al., 2002) as implemented in the FSL software (http:// www.fmrib.ox.ac.uk/fsl/). All baseline b0 images were aligned to a reference b0 image and the resulting linear transformation matrices were then applied to the diffusion-weighted images following each baseline b0 image. The gradient direction for each volume was corrected using the rotation parameters, and then the three scan repetitions were averaged to improve the signal-to-noise-ratio.

Tractography. We applied the tractographic approach as outlined by Anwander and colleagues (Friederici et al., 2006; Anwander et al., 2007). This approach is ultimately a 3D-extension of the random walk method proposed by Koch and colleagues (2002) and estimates a spatial probability distribution of connectivity from the seed regions.

Seed regions. All tractography was done in each subject's native space data (interpolated to $1 \times 1 \times 1 \mathrm{~mm}$ isotropic voxels), and resulting maps were warped into standard space (using the Montreal Neurological Institute $1 \mathrm{~mm}$ isotropic brain as reference) for cross-subject averaging and comparison. To ensure that the computed tractograms were dominated by long-range connections, seed points were placed at the gray matter/ white matter interface (white matter, fractional anisotropy $>0.15$ ). The seed and target areas were determined based on the high-resolution T1weighted anatomical scans. The aMCC as well as the thalamic regions of interest was outlined manually by an expert (S.S.) using MRicro and verified by an independent rater (M.U.). Based on prior metaanalyses of performance monitoring (Ridderinkhof et al., 2004), the aMCC seed region was chosen on the left hemisphere such that it was located at the

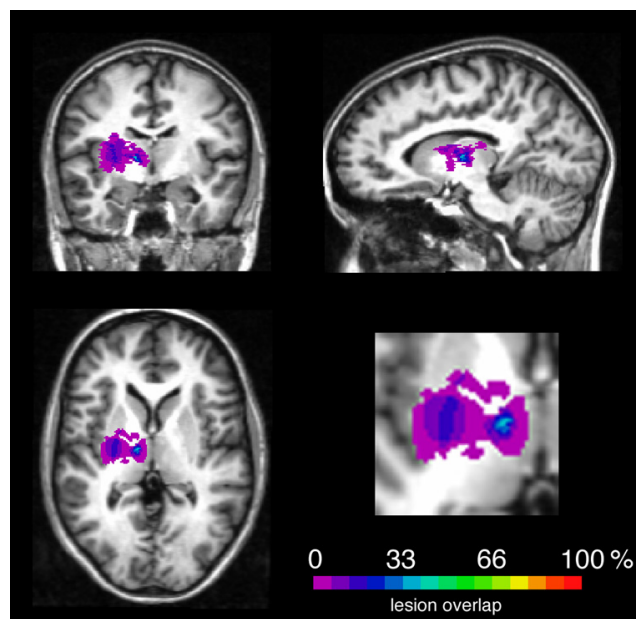

Figure 1. Overlap of the segmented lesions affecting the thalamus displayed on a healthy T1-weighted MR image. Right-hemispheric lesions were flipped to the left side.

dorsal bank of the cingulate sulcus $\sim 20 \mathrm{~mm}$ anterior to the coronal plane through the anterior commissure and had a diameter of $\sim 8 \mathrm{~mm}$. The mean volume of the aMCC seed mask was $231.43 \pm 41.60 \mathrm{~mm}^{3}$. The thalamic target masks were drawn on the left thalamus according to an atlas (Jones, 2007). Because the goal was to investigate the relative connectivity of the aMCC to the ventral anterior and ventrolateral anterior nuclei of the thalamus, the region comprising these structures formed the VA/VLa target region $\left(943.93 \pm 66.90 \mathrm{~mm}^{3}\right)$. For comparison, all other thalamic regions formed the non-VA/VLa target region $(2445.31 \pm$ $164.57 \mathrm{~mm}^{3}$ ). For each voxel in the aMCC seed masks, the number of samples reaching a particular thalamic target was recorded.

Connectivity measure. Considering all the nerve fibers originating in the source region $A$ (aMCC for this study), the structural connectivity may be defined in terms of the proportion of those fibers that intersect the target region $B$ while running within the brain white matter, yielding a number in the interval between zero (none of the fibers intercept $B$ ) and one (all fibers starting in $A$ reach $B$ ). This quantity gives no information about the absolute number of connections between two regions, but reflects the degree of connectedness or the relative connection density. The connectivity of $A$ with $B$ is direction-dependent, thus generally not identical to that of $B$ with $A$ (not to be mixed up with the direction of signal exchange, i.e., afferent and efferent fibers). To remove this dependency on the seed region, we computed the connectivity metric using each region as a seed region once and then averaging the result. If the area 


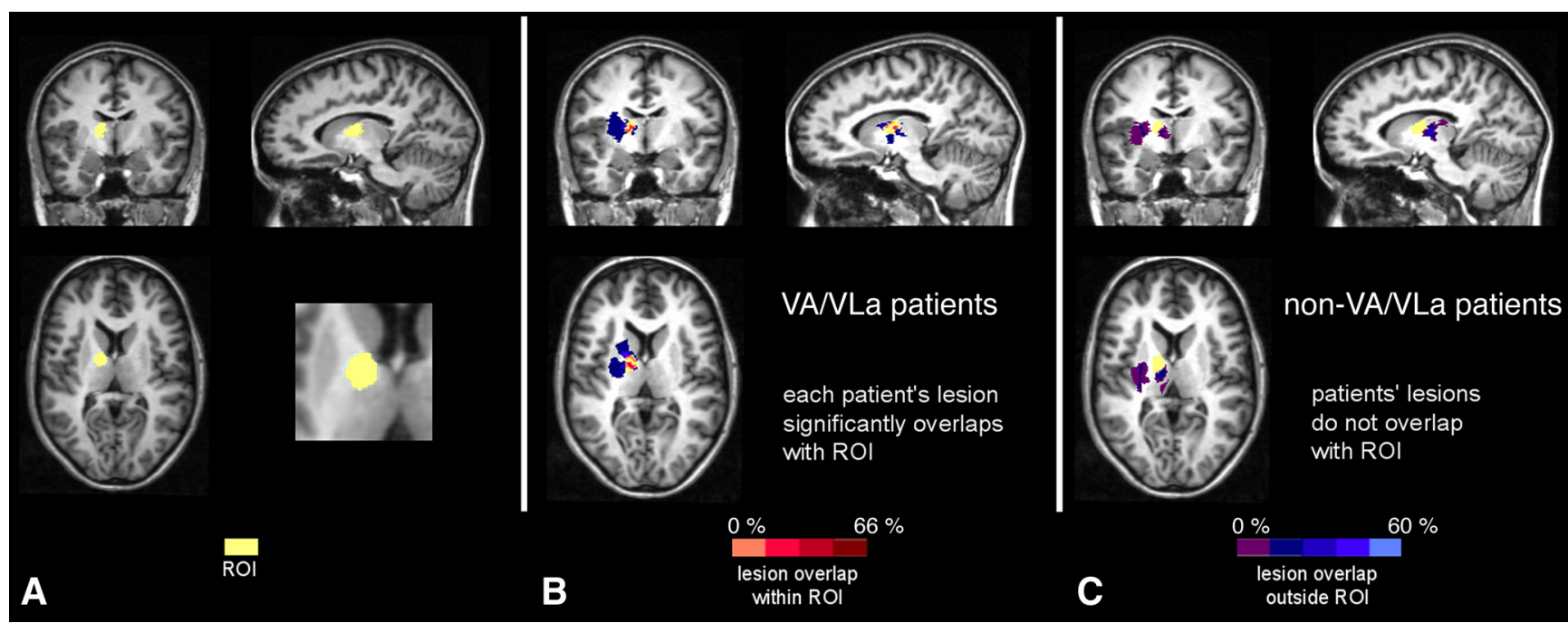

Figure 2. Separation of the patients into two subgroups according to the lesion location relative to an ROI (yellow) encompassing the VA and VLa nuclei of the thalamus. $\boldsymbol{A}$, Region of interest. $\boldsymbol{B}$, Lesion overlap of VA/VLa patients. C, Lesion overlap of non-VA/VLa patients. Hot colors indicate overlap of the lesions with the ROI, cool colors indicate overlap of the lesions outside the ROI.

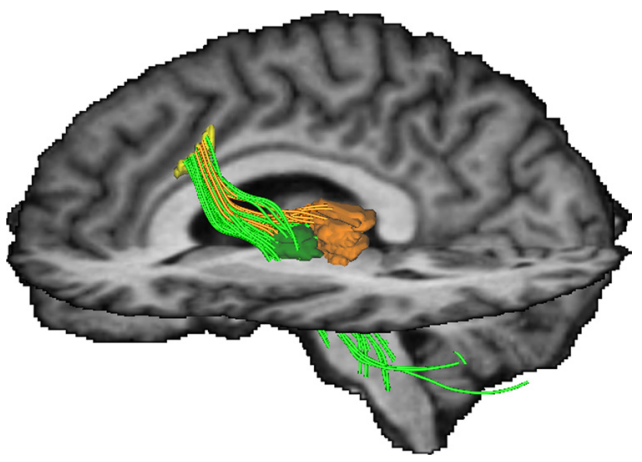

Figure 3. Representative tractogram depicting the connections between the seed region in the left rostral cingulate zone (aMCC, yellow) and the thalamic subregions (VA/VLa, green; remaining portion of the thalamus, orange). On the example of a single subject's MRI dataset, the group average seed region in the left rostral cingulate zone and target regions in the left ventral anterior and ventral lateral anterior thalamic nuclei and remaining portion of the thalamus (non-VA/VLa, brown) are shown. The connecting pathways between aMCC and thalamic regions are colored accordingly. Note that the majority of streamlines are clearly heading toward the VA/VLa regions. For visualization purposes, pathways extending in directions other than toward the thalamus have been clipped.

or volume of $A$ approaches a point, this measure reduces to the existence formulation proposed by Behrens et al. (2003), which only takes values on the discrete subset $[(0,1)]$ (for further details, see Kaden et al., 2007).

\section{Patient study}

Participants. Fifteen patients with chronic thalamic lesions (mean age, 44.7 years; range, $19-65$ years; mean education, 10.8 years; range, $10-13$ years; mean time since lesion, 3.9 years; range, $0.5-9$ years) and a control group of healthy participants (mean age, 44.4 years; range, $19-65$ years; mean education, 10.9 years; range, $10-13$ years) who were matched to patients with respect to age and education participated in the study. Demographic data and lesion descriptions are shown in Table 1 and a lesion overlay plot is shown in Figure 1.

To specify the results, we divided the patients into two groups, depending on the anatomical differences of their thalamic lesions. Therefore we defined a region of interest (ROI) as being comprised of the VA nucleus and the VLa nucleus of the thalamus, according to the atlas by Jones (2007). A group of six patients (one female) showed substantial lesion overlap with the ROI (VA/VLa group; mean age, 46.7 years; range, 27-66; mean education, 10.9 years; range, $10-13$ years; mean time since lesion, 4.7 years; range, $1-9$ years). Only one patient from this group had
Table 2. Mean proportions and reaction times of correct and erroneous responses in patients and controls broken down by compatibility

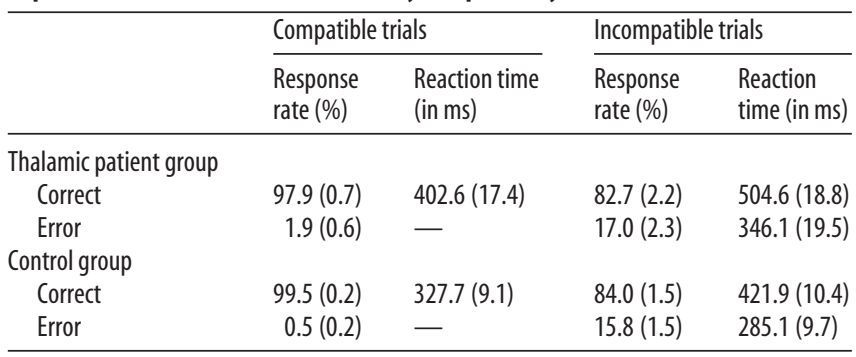

SEMs are shown in parentheses. - , The number of compatible errors was insufficient to obtain reliable reaction times for this condition.

Table 3. Mean error rates and hit reaction times for incompatible trials in patients and controls broken down by accuracy of the preceding trial

\begin{tabular}{llllll}
\hline & \multicolumn{2}{l}{ Previous correct } & & \multicolumn{2}{l}{ Previous error } \\
\cline { 2 - 3 } & $\begin{array}{llllll}\text { Error rate } \\
(\%)\end{array}$ & $\begin{array}{l}\text { Hit reaction time } \\
\text { (in ms) }\end{array}$ & & $\begin{array}{l}\text { Error rate } \\
(\%)\end{array}$ & $\begin{array}{l}\text { Hit reaction } \\
\text { time (in ms) }\end{array}$ \\
\hline Thalamic patients & $11.8(1.7)$ & $510.5(22.0)$ & & $15.1(3.5)$ & $504.4(20.9)$ \\
Control group & $13.1(1.3)$ & $416.7(9.2)$ & & $9.2(1.7)$ & $431.8(14.9)$ \\
\hline
\end{tabular}

SEMs are shown in parentheses.

an additional lesion in the MD nucleus of the thalamus. In comparison, the lesions of a second group of nine patients (four females) did not affect the ROI (non-VA/VLa group; mean age, 43.4 years; range, 19-65 years; mean education, 10.7 years; range, $10-13$ years; mean time since lesion, 3.4 years; range, $0.5-8$ years). In seven of these nine non-VA/VLa patients, the MD was clearly affected (Table 1). Age, sex, and education were matched between each patient subgroup and its control subgroup; however, patient subgroups could not be matched to each other with respect to sex. Figure 2 shows an ROI plot and the lesion overlap with the ROI and outside the ROI.

Procedure. A speeded modified flanker task known to elicit the ERN and to be suitable for patient studies was used in the study (Ullsperger and von Cramon, 2006b). Participants were instructed to respond to a target arrow, which had an onset delayed by $80 \mathrm{~ms}$ from the onset of four flanker arrows (two above and two below the target arrow) and which occurred for $110 \mathrm{~ms}$ on the screen. In 50\% of trials (960 trials, divided into four sets), the flankers pointed in the same direction as the target (compatible trial), and in the other half of the trials, the arrows pointed in the opposite direction (incompatible trial). Participants had to respond with maximal speed and accuracy to the target arrow with the hand 


\section{Thalamic Patients}
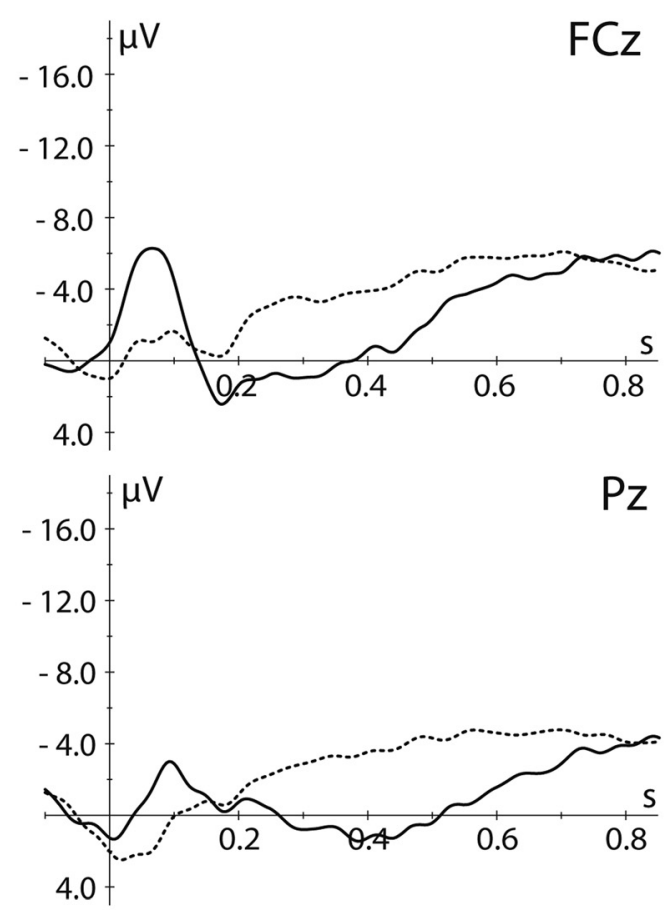

incompatible error
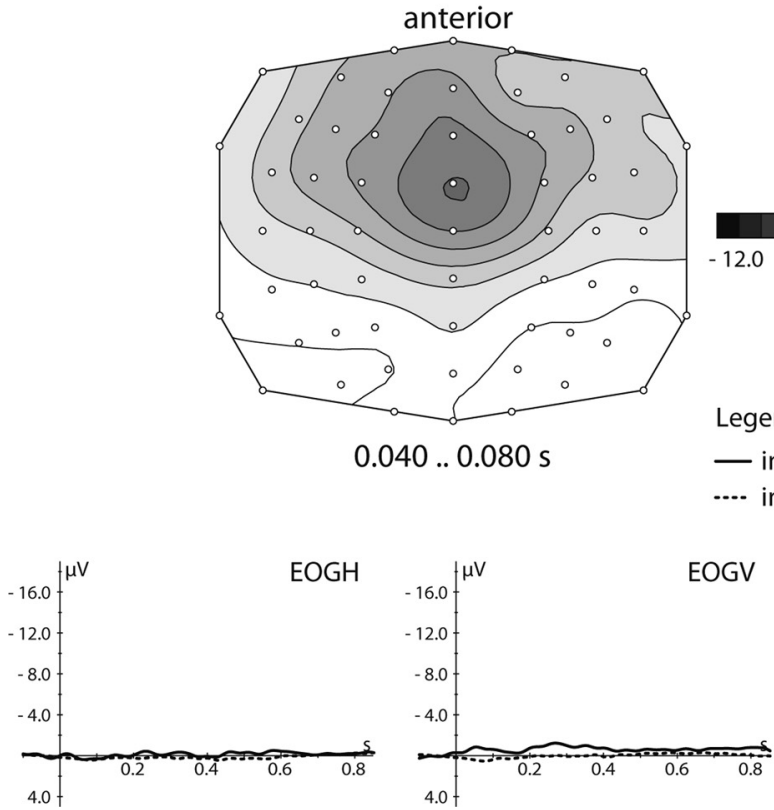

Control Group
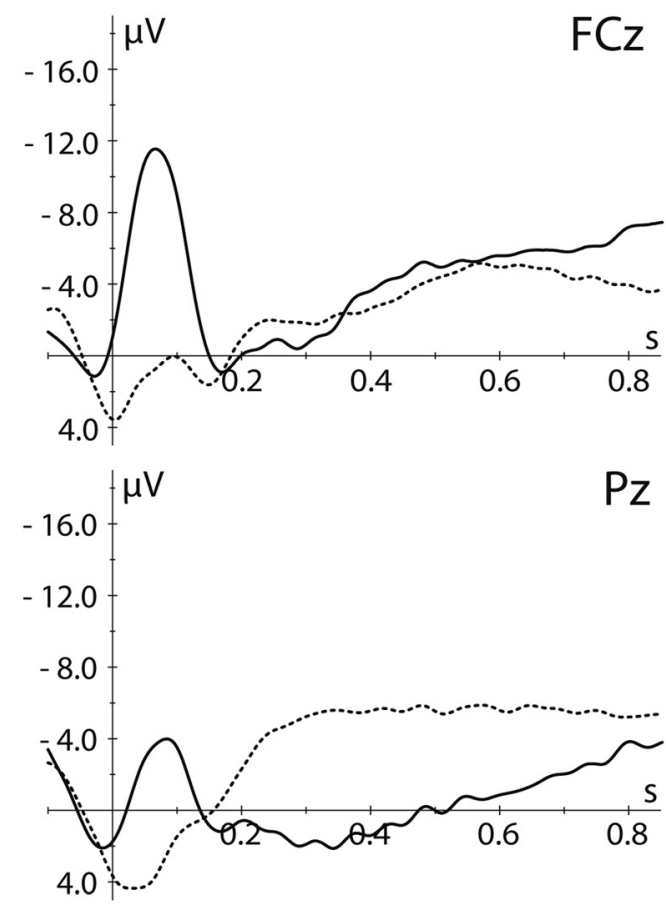

incompatible error

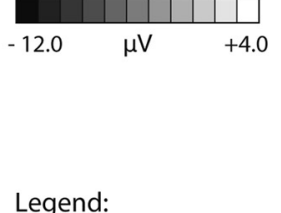

Legend:

— incompatible error

.... incompatible correct
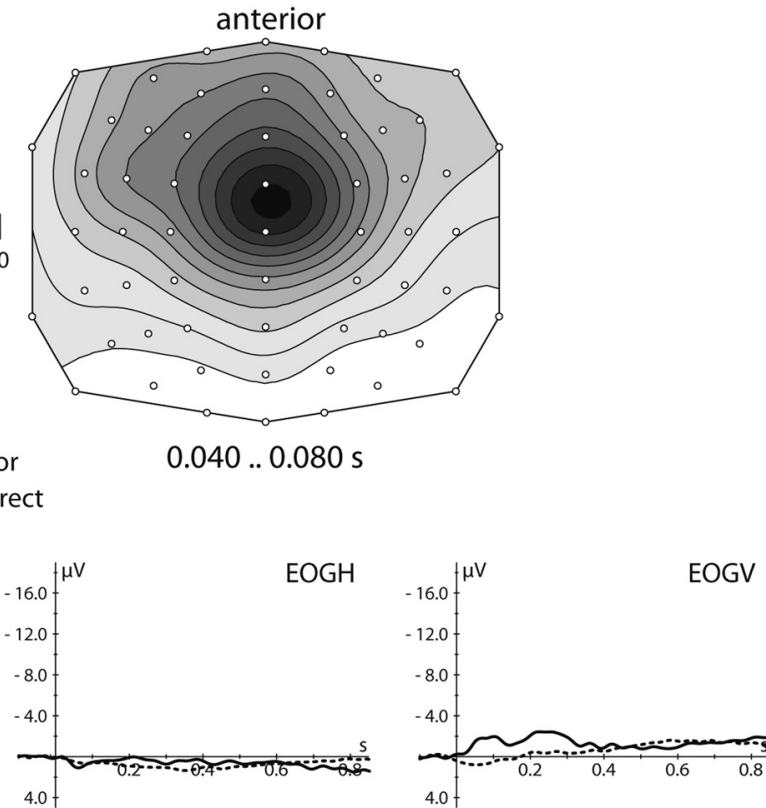

Figure 4. Results of the performance-monitoring study in patients with thalamic lesions. Top, Response-locked grand mean average waveforms of the event-related potentials to errors (solid line) and correct responses (dotted line) shown for patients (left) and controls (right) at two midline electrodes. Middle, Topographical distribution of the error-related negativity over the scalp shown for patients (left) and controls (right). Bottom, Grand mean waveforms at ocular electrodes after artifact correction. EOGH, Horizontal electrooculogram; EOGV, vertical electrooculogram.

indicated by its direction. If they noticed an error response, participants were instructed to press a third button in the center of the keyboard within $1400 \mathrm{~ms}$ after target onset (Ullsperger and von Cramon, 2006a). Additionally, we made use of an adaptive algorithm that dynamically adjusted the response-time pressure based on the participants' performance, and aimed at an optimization of error rate by $20 \%$ on incompatible errors (Fiehler et al., 2005).

Electrophysiological recordings. The participants were seated comfortably in a dimly lit, electrically shielded chamber. The electroencephalo- gram (EEG) was recorded with $\mathrm{Ag} / \mathrm{AgCl}$ electrodes from a 64-channel EEG system (BrainAmps MR+; BrainProducts), referenced to left mastoid and offline re-referenced to the arithmetic mean of both mastoids. Electrode impedance was kept $<5 \mathrm{k} \Omega$. The vertical electrooculogram (EOG) was recorded from electrodes placed above and below the right eye. To monitor horizontal eye movements, the EOG was collected from electrodes placed on the outer canthus of the left and right eye. EEG and EOG were recorded continuously with a low pass filter of $70 \mathrm{~Hz}$, and analog-to-digital converted with 16-bit resolution at a sampling rate of 
$250 \mathrm{~Hz}$. First, the EEG epochs were scanned for muscular and other large artifacts. Whenever the SD in a $200 \mathrm{~ms}$ interval exceeded $50 \mu \mathrm{V}$, the epoch was rejected. Second, horizontal and vertical EOG artifacts present in the EEG signal were corrected by an eye-movement correction procedure, based on a linear regression method as described previously (Gratton et al., 1983).

Data analysis. The time difference between target onset and the button press was defined as response time. When an error was signaled by a subsequent press of the signaling button, the time difference between the first (erroneous) and the second (signaling) response was defined as signaling time. When participants corrected an error spontaneously by pressing the correct key, the time difference was defined as spontaneous correction time. Responses were analyzed when they occurred within $2000 \mathrm{~ms}$ of target onset. To test for posterror trial-by-trial adjustments, error rates and response times were submitted to ANOVAs with the factors previous response type (two levels) and group. To control for confounding conflict adaptation effects (Ullsperger et al., 2005), the analysis was restricted to incompatible hits preceded by incompatible hits or errors.

Response-locked ERP epochs were averaged separately for incompatible correct and incompatible erroneous trials, starting $100 \mathrm{~ms}$ before the response and continuing $500 \mathrm{~ms}$ after the response. Compatible trials were excluded from ERP analyses because of an insufficient number of error trials $(<1 \%)$, as were responses delivered after the response deadline. The average voltage in the $100 \mathrm{~ms}$ preceding the onset of the flanker arrows served as baseline.

For the quantification of the ERN, peak-to-peak measurements were calculated to determine baseline-independent amplitudes of negative deflections by subtracting the amplitude of the preceding positive peak from the negative peak of this component (Falkenstein et al., 2000). Based on the literature, time search windows of the ERN were chosen a priori. Two early time windows were defined from -60 to $0 \mathrm{~ms}$ for the positive peak preceding the ERN, and from 0 to $140 \mathrm{~ms}$ for the ERN component. To analyze the error positivity (Pe) (Falkenstein et al., 1990), the mean amplitude between 300 and $500 \mathrm{~ms}$ was used.

Statistical effects were determined at representative electrodes where the ERN and Pe are largest (F3, FCz, F4, C3, Cz, C4, P3, Pz, P4) by means of repeated-measures ANOVA with the between-subjects factor group (all thalamic patients, controls, VA/VLa group, non-VA/VLa group) and the within-subjects factors response type (correct, incorrect) and two topographical factors (anterior-posterior dimension: frontal, central, parietal; lateral dimension: left, midline, right). All effects with more than one degree of freedom in the numerator were adjusted for violations of sphericity according the formula of Huynh and Feldt (1970). To avoid reporting large amounts of statistical results not relevant to the issues under investigation, only main effects or interactions, including the factors response type and group, are reported here. Topographical scalp potential maps were generated using a two-dimensional spherical spline interpolation and a radial projection from $\mathrm{Cz}$, which respects the length of the median arcs. For graphical display, a low-pass filter with a cutoff frequency of $15 \mathrm{~Hz}$ was applied. Results are listed as mean \pm SEM unless otherwise specified.

Lesion data analysis. The lesions of the patients and the region of interest were segmented manually with MRIcro based on high-resolution 3D T1-weighted anatomical MR datasets. These patient datasets were aligned and normalized to standard stereotactic space (Talairach and Tournoux, 1988) by affine transformation. The rotational and translational parameters were subsequently used to transform lesion segments using trilinear interpolation, such that the resulting segments were aligned with the stereotactic coordinate system. Datasets for patients with lesions in the right hemisphere (patient IDs: P789, P845, P1082, P1298) were flipped to allow lesion overlap analyses. For visualization purposes, the lesion data of the patients were overlapped for each patient group to form density maps (Rorden and Karnath, 2004).

\section{Results}

\section{Diffusion-based imaging}

Diffusion-based magnetic resonance imaging has been used to derive a structural description of the connectedness of brain re-
Table 4. Results of ERP amplitude analyses for the ERN and Pe

\begin{tabular}{|c|c|c|c|c|c|c|}
\hline \multirow[b]{2}{*}{ Variance } & \multicolumn{3}{|l|}{ ERN } & \multicolumn{3}{|l|}{$\mathrm{Pe}$} \\
\hline & df & $F$ & $p$ & $d f$ & $F$ & $p$ \\
\hline Group & 1,28 & 5.00 & $<0.05$ & 1,28 & 0.63 & $>0.43$ \\
\hline Group $\times$ Resp & 1,28 & 6.43 & $<0.05$ & 1,28 & 0.14 & $>0.72$ \\
\hline Group $\times \operatorname{Resp} \times A P$ & 2,56 & 4.90 & $<0.05$ & 2,56 & 11.4 & $<0.001$ \\
\hline Group $\times$ Resp $\times$ Lat & 2,56 & 2.13 & 0.13 & 2,56 & 3.48 & $<0.05$ \\
\hline $\begin{array}{l}\text { Group } \times \text { Resp } \times \text { AP } \times \text { Lat } \\
\text { Incompatible correct response }\end{array}$ & \multicolumn{5}{|c|}{ Incompatible correct response } & $<0.05$ \\
\hline Group & 1,28 & 0.12 & $>0.7$ & 1,28 & 0.14 & $>0.72$ \\
\hline Group $\times A P \times$ Lat & 4,112 & 0.55 & $>0.65$ & 4,112 & 0.82 & $>0.47$ \\
\hline \multicolumn{7}{|l|}{ Incompatible erroneous response } \\
\hline Group & 1,28 & 8.98 & $<0.005$ & 1,28 & 0.89 & $>0.36$ \\
\hline Group $\times$ AP $\times$ Lat & 4,112 & 10.50 & $<0.001$ & 4,112 & 1.79 & $>0.17$ \\
\hline \multicolumn{7}{|l|}{ Control group } \\
\hline Resp & 1,14 & 41.21 & $<0.001$ & 1,14 & 4.67 & $<0.05$ \\
\hline $\operatorname{Resp} \times A P$ & 2,28 & 11.28 & $<0.005$ & 2,28 & 12.3 & $<0.005$ \\
\hline Resp $\times$ Lat & 2,28 & 4.90 & $<0.05$ & 2,28 & 17.64 & $<0.001$ \\
\hline Resp $\times$ AP $\times$ Lat & 4.56 & 22.70 & $<0.001$ & 4.56 & 6.37 & $<0.005$ \\
\hline \multicolumn{7}{|l|}{ Thalamic patient group } \\
\hline Resp & 1,14 & 15.69 & $<0.005$ & 1,14 & 5.52 & $<0.05$ \\
\hline $\operatorname{Resp} \times A P$ & 2,28 & 1.25 & $>0.3$ & 2,28 & 2.16 & $>0.16$ \\
\hline Resp $\times$ Lat & 2,28 & 3.28 & $<0.1$ & 2,28 & 8.01 & $<0.01$ \\
\hline Resp $\times A P \times$ Lat & 4.56 & 5.18 & $<0.01$ & 4.56 & 1.86 & $>0.17$ \\
\hline
\end{tabular}

The upper part of the table shows the results of the omnibus ANOVA; below that, the results of planned comparisons are broken down by the factors response and group. Effects and interactions not listed are not significant. Resp, Response factor; AP, anterior-posterior factor; Lat, lateral factor; df, degrees of freedom.

gions in vivo (Johansen-Berg and Rushworth, 2009), including aMCC (Beckmann et al., 2009) and thalamus (Behrens et al., 2003). As we here wish to quantify anatomical connectivity between distant brain regions, which may not just be plain voxels (Koch et al., 2002) or single points (e.g., the center of voxels) (Behrens et al., 2003; Parker et al., 2003), we followed the more generalized definition used by Kaden and colleagues (2007), who defined the anatomical connectivity as the proportion of fiber pathways, originating in a specific source region, that intersect a target region, thus extending the idea of connectivity to arbitrarily chosen areas or volumes. The relative strength of connectivity of the aMCC seed region with the thalamic VA/VLa target region of the thalamus (mean connection density \pm SEM, $0.04104 \pm 0.01288$ ) was significantly higher than with other thalamic regions (non-VA/VLa target region, $0.01836 \pm 0.00720$ ), as revealed by a Wilcoxon signed rank test $(p<0.0113)$. This supports the view that the major inputs from the basal ganglia loops to the aMCC are, similar to nonhuman primates, mediated via the ventral anterior and ventrolateral anterior thalamus. A representative tractogram depicting the connections between the aMCC and the thalamic regions is shown in Figure 3. Note that the fiber connections to the non-VA/VLa region appear to terminate in the area of the MD nucleus.

\section{Functional role of the thalamus in performance monitoring}

Fifteen patients with chronic focal lesions of the thalamus and a matched healthy control group underwent electroencephalography while performing a speeded flanker task suited for patient studies and known to elicit a sufficient number of errors (Ullsperger and von Cramon, 2006b). Behaviorally, both patients and controls showed equivalent flanker interference effects typical for flanker tasks, i.e., longer response times and higher error rates for incompatible trials than for compatible trials (main effect of compatibility, reaction times, $F_{(1,28)}=692.41, p<0.0001$; error rates, $F_{(1,28)}=118.45, p<0.0001$; no group effect, $p>0.37$ ) (Tables 2 and 3 ). Importantly, resulting from individual adjustments of time pressure, the rate of incompatible errors, i.e., the 
events under investigation, did not differ significantly between groups (patients, $17.0 \pm 1.8 \%$, vs controls, $15.8 \pm 1.8 \%$; $p>0.64)$. Compared with the controls, the thalamic patients generally had prolonged reaction times $\left(F_{(1,28)}=15.20, p<\right.$ 0.001 ).

To test error detection, participants were instructed to indicate encountered errors by pressing an error-signaling button (Ullsperger and von Cramon, 2006a). Error signaling was significantly diminished and delayed in the patients (mean \pm SEM, $39.1 \pm 10.7 \%$, $972 \pm 128 \mathrm{~ms})$ compared with the controls $\left(85.1 \pm 6.9 \%, t_{(28)}=3.83\right.$, $p<0.001 ; 708 \pm 56 \mathrm{~ms}, t_{(28)}=2.16, p<$ 0.05). In contrast, spontaneous errorcorrection time and spontaneous correction rate for both groups did not differ significantly (patients, $266 \pm 48 \mathrm{~ms}, 30.3 \pm 8.4 \%$; controls, $183 \pm 23 \mathrm{~ms}, 20.7 \pm 4.3 \% ; p>$ 0.21 ), supporting the notion that error correction is not a sensitive measure of error processing (Rabbitt, 2002; Ullsperger and von Cramon, 2006a). In $42.3 \pm 10.4 \%$ of erroneous responses, no second key press was recorded for the patients at all, compared with the control group $\left(8.4 \pm 5.9 \%, t_{(28)}=\right.$ $2.82, p<0.01)$. This verifies that the patients responded less adequately to errors than their controls.

The control group showed significant slowing of reactions on trials subsequent to errors $\left(F_{(1,14)}=7.90, p<0.05\right)$, which was accompanied by posterror improvement in accuracy $\left(F_{(1,14)}=5.08, p<0.05\right)$. In contrast, the patients' reduced error-detection ability was accompanied by abolished posterror slowing $(p>0.91)$ (Tables 2 and 3$)$. Moreover, the patients showed no significant change in error likelihood on posterror trials $(p>0.39)$. These results show that patients with thalamic lesions were impaired in detecting errors and adjusting behavior accordingly.

These behavioral findings were accompanied by significant group differences in the ERN (Fig. 4). In the control group, errors elicited a typical ERN, reaching a trough-to-peak amplitude of $-11.5 \pm 0.9 \mu \mathrm{V}$ at $\mathrm{FCz}$, and a posteriorly located Pe 300-500 ms after the erroneous response. In contrast, patients showed a significantly reduced ERN amplitude of $-6.6 \pm 0.8 \mu \mathrm{V}$ and a diminished Pe (for detailed statistical results, see Table 4). No group difference was found between ERP waveforms and correct responses $(p>0.47)$.

To test the specific functional contribution of the VA/VLa nuclei to performance monitoring, the ERP data were analyzed for two patient subgroups, the VA/VLa group (comprised of six patients in whom lesions affected the VA/VLa region of the thalamus) and the non-VA/VLa group (comprised of nine patients in whom these nuclei remained unaffected) (Fig. 5). Patients from the non-VA/VLa group showed a significant ERN (main effect of response, $F_{(1,08)}=33.65, p<0.001$; interaction response $\times$ topographical factors, $\left.F_{(4,32)}=6.62, p<0.01\right)$, though of reduced amplitude compared with the according controls (interaction response $\times$ topographical factors $\times$ group, $F_{(4,64)}=3.43, p<$ $0.05)$. In contrast, in VA/VLa patients, no significant amplitude difference between the ERPs for correct and incorrect trials were found at the ERN latency range ( $p s>0.43$ ). Compared with their controls, there was a significant difference between ERPs in the
VA/VLa

non VA/VLa

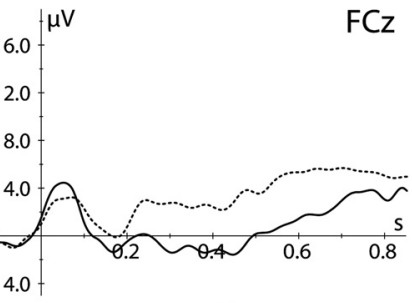

- incompatible error

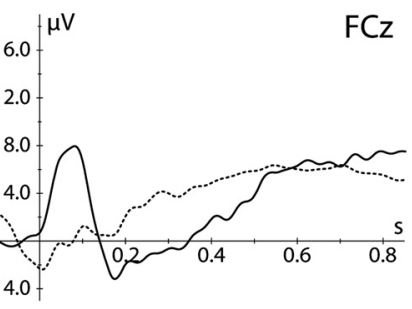

B

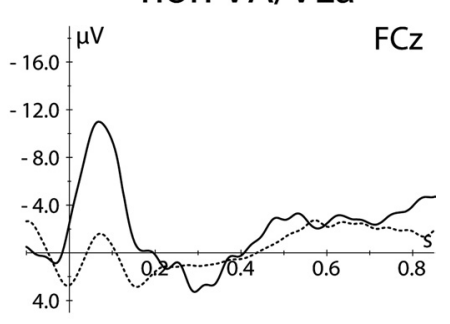

C

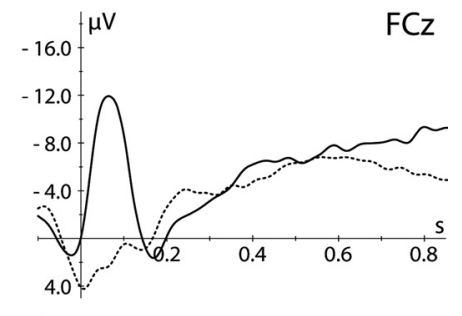

D

Figure 5. Differential effect of thalamic lesions on ERN. $\boldsymbol{A}-\boldsymbol{D}$, Response-locked grand mean average event-related potential waveforms for errors (solid line) and correct responses (dotted line) at electrode FCz shown for patients with lesions in the VA/VLa nuclei of the thalamus $(\boldsymbol{A})$, their controls $(\boldsymbol{B})$, patients with other thalamic lesions (non-VA/VLa, $\boldsymbol{C}$ ), and their controls $(\boldsymbol{D})$.

Table 5. Results of statistical ERP analyses for incompatible trials restricted to patients with either VA/VLa or MD lesions and their controls

\begin{tabular}{|c|c|c|c|c|c|c|}
\hline \multirow[b]{2}{*}{ Variance } & \multicolumn{3}{|c|}{ Incompatible error } & \multicolumn{3}{|c|}{ Incompatible correct } \\
\hline & df & $F$ & $p$ & df & $F$ & $p$ \\
\hline \multicolumn{7}{|c|}{ VA/VLa +/MD - versus controls } \\
\hline Group & 1,8 & 12.14 & $<0.01$ & 1,8 & 0.0 & $>0.94$ \\
\hline Group $\times A P$ & 2,16 & 8.12 & $<0.01$ & 2,16 & 1.1 & $>0.34$ \\
\hline Group $\times$ AP $\times$ Lat & 4,32 & 3.12 & $<0.05$ & 4,32 & 0.74 & $>0.57$ \\
\hline \multicolumn{7}{|c|}{ VA/VLa $-/ M D+$ versus controls } \\
\hline Group & 1,12 & 3.96 & 0.07 & 1,12 & 0.92 & $>0.35$ \\
\hline Group $\times P \times$ Lat & 4,48 & 8.32 & $<0.001$ & 4,48 & 1.21 & $>0.31$ \\
\hline \multicolumn{7}{|c|}{ VA/VLa $+/ M D-$ versus VA/VLa $-/ M D+$} \\
\hline Group & 1,10 & 10.44 & $<0.01$ & 1,10 & 0.37 & $>0.55$ \\
\hline Group $\times A P$ & 2,20 & 2.71 & $<0.1$ & 2,20 & 0.7 & $>0.47$ \\
\hline
\end{tabular}

Effects and interactions not listed are not significant. AP, Anterior-posterior factor; df, degrees of freedom; VA/ $\mathrm{VLa}+/ \mathrm{MD}-$, patients with lesions to VA/VLa but not MD; VA/VLa $-/ M D+$, patients with lesions to MD but not VA/VLa.

Table 6. Results of statistical ERP analyses for incompatible trials restricted to patients with either VA/VLa or MD lesions and their controls

\begin{tabular}{|c|c|c|c|c|c|c|}
\hline \multirow[b]{2}{*}{ Variance } & \multicolumn{3}{|c|}{ VA/VLa +/MD- } & \multicolumn{3}{|c|}{ VA/VLa-/MD+ } \\
\hline & $d f$ & $F$ & $p$ & df & $F$ & $p$ \\
\hline Resp & 1,4 & 1.51 & $>0.28$ & 1,6 & 18.93 & $<0.005$ \\
\hline $\operatorname{Resp} \times A P$ & 2,8 & 0.86 & $>0.41$ & 2,12 & 2.85 & $<0.1$ \\
\hline Resp $\times$ Lat & 2,8 & 0.11 & $>0.87$ & 2,12 & 4.59 & $<0.05$ \\
\hline $\operatorname{Resp} \times A P \times$ Lat & 4,16 & 0.58 & $>0.59$ & 4,24 & 4.01 & $<0.05$ \\
\hline
\end{tabular}

Effects and interactions not listed are not significant. Resp, Response factor; AP, anterior-posterior factor; Lat, lateral factor; df, degrees of freedom; VA/VLa +/MD - , patients with lesions to VA/VLa but not MD; VA/VLa - / $M D+$, patients with lesions to MD but not VA/VLa.

ERN time range for incorrect trials (group effect, $F_{(1,28)}=8.98$, $p<0.01$; two-way and triple interactions group $\times$ topographical factors, $F s>4.82$, ps $<0.05)$ but not for correct trials $(F s<1.6$, ps $>0.21)$. In other words, the ERN is abolished in patients whose lesions substantially overlap with the ventral anterior and ventrolateral anterior thalamus.

In addition to the VA and VLa, the MD nucleus of the thalamus has been shown to project to the aMCC (Vogt et al., 1979; Baleydier and Mauguiere, 1980; Hatanaka et al., 2003). To differ- 


\section{A Thalamic Patients}
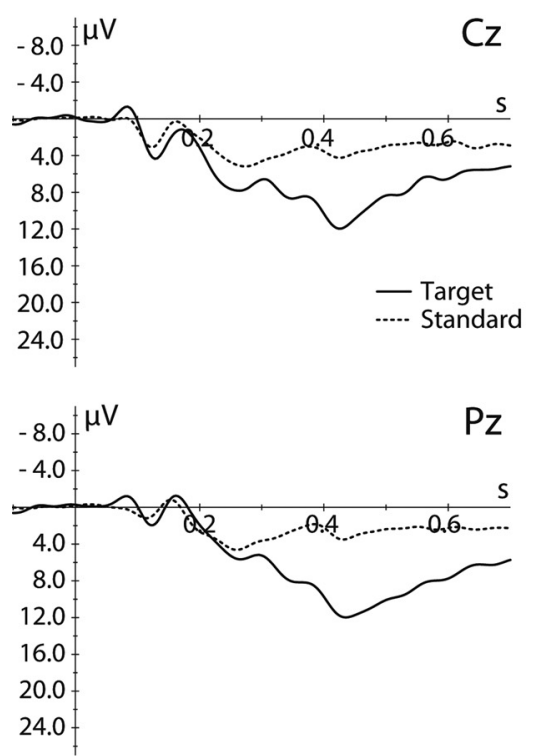

C VA/VLa Group
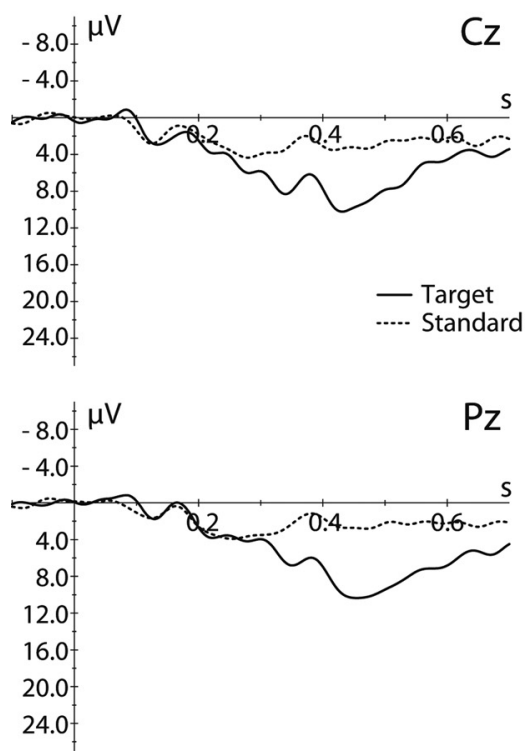

\section{B Control Group}
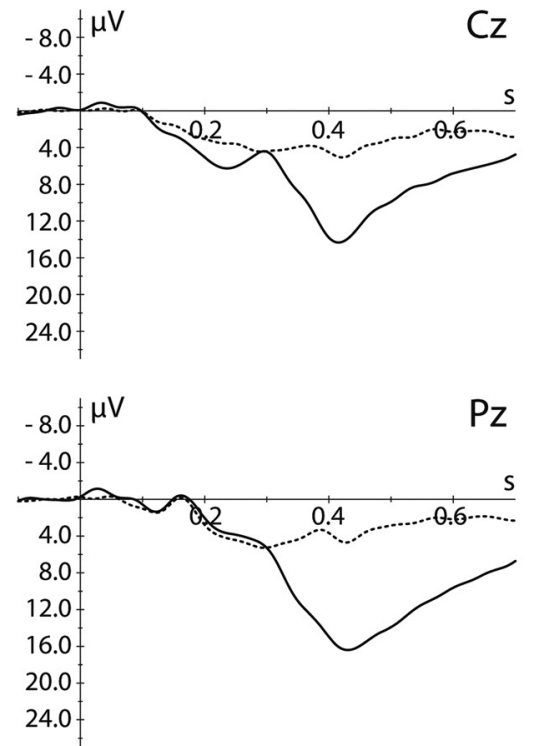

D non VA/VLa Group
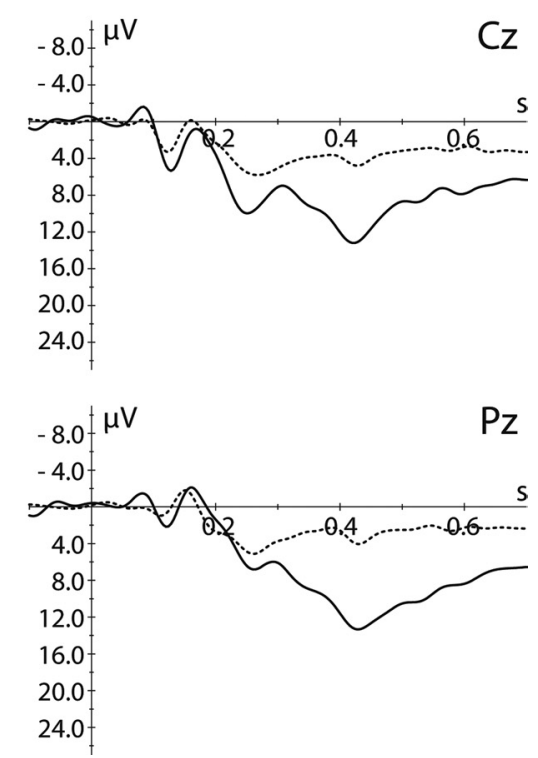

Figure 6. $\quad \boldsymbol{A}, \boldsymbol{B}$, Stimulus-locked grand mean average waveforms for target (solid line) and standard (dotted line) stimuli in the oddball task shown at two midline electrodes separately for the thalamic patients $(\boldsymbol{A})$ and control group $(\boldsymbol{B})$. $\boldsymbol{C}, \boldsymbol{D}$, Data for the VA/VLa ( $)$ and non-VA/VLa subgroups (D).

entiate the functional significance of the MD in performance monitoring, we compared the ERN data of all VA/VLa patients without additional MD lesion (five of six patients) (Table 1) and all non-VA/VLa patients with clear MD lesion (seven of nine patients) with each other and to their healthy controls. This restriction of the patient groups specified that VA/VLa lesions were associated with a loss of the ERN, whereas this component was present and only somewhat smaller in thalamic lesions affecting the MD but sparing VA/VLa (for detailed statistics, see Tables 5 and 6).

\section{Specificity of lesion effect to performance monitoring}

To test whether thalamic lesions have a general detrimental effect on ERPs, we investigated the P300 for patients and controls in a visual oddball task in which participants had to press a response button whenever a rare visual target stimulus was presented in a sequence of standard visual stimuli. For target responses, the waveform of both groups showed a clear centroparietal P300 (Fig. 6). Neither amplitude nor topography differed significantly between thalamic patients and healthy controls or between thalamic subgroups and their controls ( $p s>0.33$ ), suggesting that thalamic lesions did not have a general effect on cognitive ERPs.

\section{Discussion}

These data show that the ventral anterior and ventrolateral anterior thalamus is anatomically and functionally closely connected with the aMCC. Diffusion-based tractography confirms the notion based on anatomical studies in animals that, among the thalamic nuclei, the VA/VLa nuclei have relatively strong connectivity with the aMCC in humans. Notably, diffusion-based connectivity measures provide neither direction information nor an absolute number of fiber connections, but they are highly useful for the comparison of relative connectivity strength between cortical and subcortical regions. Furthermore, it should be noted that the ROI encompassing VA/VLa may have included small portions of adjacent anterior nuclei and the internal capsule, such that some connections from anterior thalamic nuclei might have contributed to the findings. Moreover, the data also suggest relatively strong connections from the MD to aMCC, which is in line with previous findings in monkeys (Vogt et al., 1979; Baleydier and Mauguiere, 1980; Barbas et al., 1991; Hatanaka et al., 2003).

The patient data suggest that the thalamus, particularly its VA/VLa region, is functionally necessary for performance monitoring. Thalamic lesions are associated with an impairment of ERN generation. The subgroup data suggest a gradient in impairment ranging from slight amplitude reduction in posterior and medial lesions affecting the MD to complete loss of the ERN in ventrolateral anterior and ventral anterior lesions. In contrast to previous patient studies, in which an absent ERN was not always accompanied by unequivocal behavioral deficits (in part because less sensitive measures than error detection, such as error correction, were examined) (Ullsperger and von Cramon, 2006a), here the patients showed clear behavioral performance-monitoring impairment. In particular, conscious error detection, as indicated by the error-signaling procedure, was reduced in the patients, showing that they were aware of less than half of the committed errors, whereas healthy controls noticed almost all errors. In addition, in contrast to the controls, patients showed no trial-by-trial adjustments, such as posterror slowing (Rabbitt, 1966) and posterror improvement in accuracy. The concomitant abolishment of the ERN and posterror behav- 
ioral adjustments in the patients support the view that the aMCC signals the need for adaptation. This is consistent with findings that EEG and hemodynamic correlates of aMCC activity predict posterror slowing (Garavan et al., 2002; Kerns et al., 2004; Debener et al., 2005; Klein et al., 2007; Cavanagh et al., 2009; King et al., 2010; Wessel and Ullsperger, 2011). Thus, thalamocingulate interactions appear to be necessary for the generation of the error signal in the aMCC, reflected in the ERN, and its use for efficient initiation of motor inhibition is thought to underlie posterror slowing and top-down attentional focusing (King et al., 2010).

It should be noted that, in many patients, the lesions were not completely confined to the thalamus, but also affected neighboring structures, such as the internal capsule and the putamen. Striatal lesions have been shown to affect the ERN (Ullsperger and von Cramon, 2006b). Notably, the region-of-interest analysis of the VA/VLa area shows that the electrophysiological findings are specific to the frontostriato-thalamocortical circuits connected via these predominantly motor-related thalamic nuclei. Importantly, all behavioral and ERP findings remained significant when a patient with an additional lesion of the lateral prefrontal cortex (P325) was excluded from analysis, further supporting the specificity of the results to the thalamus.

This study particularly focused on the thalamic VA/VLa nuclei, as this region connects motor-action-related striatal and cerebellar inputs with the motor-related rostral cingulate zone in aMCC (Van Hoesen et al., 1993; Hatanaka et al., 2003; Hoshi et al., 2005), monitoring of which, according to current models of performance, should be relevant for detecting deviations in action outcomes (Holroyd and Coles, 2002). Notably, other regions of the thalamus are connected with the aMCC as well, in particular the MD and anterior and intralaminar nuclei, implicated in pain processing (Vogt et al., 1993; Vogt, 2005), arousal, and awareness (Van der Werf et al., 2002). This is reflected in two findings of the present experiments. First, diffusion-based tractography revealed substantial, though relatively less, connectivity of the aMCC and the thalamic areas not captured by the VA/VLa region, in particular the MD. Second, patients with thalamic lesions not affecting the VA/VLa nuclei still show a somewhat reduced ERN amplitude, suggesting that input from other thalamic areas to the aMCC are of importance as well. In addition, their ability to consciously perceive errors appears similarly impaired as in the VA/VLa patients, which may have to do with the role of the MD and intralaminal nuclei in arousal and awareness.

In summary, in humans, the thalamus closely interacts with the aMCC. This interaction is of great functional importance for performance monitoring and subsequent adjustments. The performance-monitoring function appears to be represented more in the ventral anterior and ventrolateral anterior than in medial and posterior regions of the thalamus. The VA/VLa nuclei seem to modulate aMCC activity and to integrate inputs from the striatum, the lateral prefrontal cortex (via nonreciprocal corticothalamic fibers), and the cerebellum. An important aim for future research is to examine the relative contributions of these inputs for performance monitoring and cognitive control. The present findings may also be of potential clinical interest as deep brain stimulation (DBS) in nearby thalamic regions has recently been used for the treatment of Tourette's syndrome (Servello et al., 2008,2009 ). It will be important to see whether and how the impaired performance monitoring in this disorder (Johannes et al., 2002; Müller et al., 2003), reflected in increased ERN amplitudes, is modulated by DBS in the thalamus; moreover, the implantation of depth electrodes may allow direct recording of electrical activity in these regions (Marceglia et al., 2010) and link it to performance monitoring and other cognitive functions.

\section{References}

Anwander A, Tittgemeyer M, von Cramon DY, Friederici AD, Knösche TR (2007) Connectivity-based parcellation of Broca's area. Cereb Cortex $17: 816-825$.

Baleydier C, Mauguiere F (1980) The duality of the cingulate gyrus in monkey: neuroanatomical study and functional hypothesis. Brain 103:525-554.

Barbas H, Henion TH, Dermon CR (1991) Diverse thalamic projections to the prefrontal cortex in the rhesus monkey. J Comp Neurol 313:65-94.

Beckmann M, Johansen-Berg H, Rushworth MF (2009) Connectivity-based parcellation of human cingulate cortex and its relation to functional specialization. J Neurosci 29:1175-1190.

Behrens TE, Johansen-Berg H, Woolrich MW, Smith SM, Wheeler-Kingshott CA, Boulby PA, Barker GJ, Sillery EL, Sheehan K, Ciccarelli O, Thompson AJ, Brady JM, Matthews PM (2003) Non-invasive mapping of connections between human thalamus and cortex using diffusion imaging. Nat Neurosci 6:750-757.

Beste C, Saft C, Andrich J, Gold R, Falkenstein M (2006) Error processing in Huntington's disease. PLoS One 1:e86.

Beste C, Saft C, Konrad C, Andrich J, Habbel A, Schepers I, Jansen A, Pfleiderer B, Falkenstein M (2008) Levels of error processing in Huntington's disease: a combined study using event-related potentials and voxel-based morphometry. Hum Brain Mapp 29:121-130.

Cavanagh JF, Cohen MX, Allen JJ (2009) Prelude to and resolution of an error: EEG phase synchrony reveals cognitive control dynamics during action monitoring. J Neurosci 29:98-105.

Coles MG, Scheffers MK, Holroyd CB (2001) Why is there an ERN/Ne on correct trials? Response representations, stimulus-related components, and the theory of error-processing. Biol Psychol 56:173-189.

Debener S, Ullsperger M, Siegel M, Fiehler K, von Cramon DY, Engel AK (2005) Trial-by-trial coupling of concurrent electroencephalogram and functional magnetic resonance imaging identifies the dynamics of performance monitoring. J Neurosci 25:11730-11737.

Dum RP, Strick PL (1993) Cingulate motor areas. In: Neurobiology of cingulate cortex and limbic thalamus (Vogt BA, Gabriel M, eds), pp 415441. Boston: Birkhäuser.

Falkenstein M, Hohnsbein J, Hoormann J, Blanke L (1990) Effects of errors in choice reaction tasks on the ERP under focused and divided attention. In: Psychophysiological Brain Research (Brunia CHM, Gaillard AWK, Kok A, eds), pp 192-195: Tilburg UP.

Falkenstein M, Hoormann J, Christ S, Hohnsbein J (2000) ERP components on reaction errors and their functional significance: a tutorial. Biol Psychol 51:87-107.

Falkenstein M, Hielscher H, Dziobek I, Schwarzenau P, Hoormann J, Sunderman B, Hohnsbein J (2001) Action monitoring, error detection, and the basal ganglia: an ERP study. Neuroreport 12:157-161.

Fiehler K, Ullsperger M, von Cramon DY (2005) Electrophysiological correlates of error correction. Psychophysiology 42:72-82.

Frank MJ (2005) Dynamic dopamine modulation in the basal ganglia: a neurocomputational account of cognitive deficits in medicated and nonmedicated Parkinsonism. J Cogn Neurosci 17:51-72.

Frank MJ, Seeberger LC, O’Reilly RC (2004) By carrot or by stick: cognitive reinforcement learning in parkinsonism. Science 306:1940-1943.

Frank MJ, Samanta J, Moustafa AA, Sherman SJ (2007) Hold your horses: impulsivity, deep brain stimulation, and medication in parkinsonism. Science 318:1309-1312.

Friederici AD, Bahlmann J, Heim S, Schubotz RI, Anwander A (2006) The brain differentiates human and non-human grammars: functional localization and structural connectivity. Proc Natl Acad Sci U SA 103:2458-2463

Garavan H, Ross TJ, Murphy K, Roche RA, Stein EA (2002) Dissociable executive functions in the dynamic control of behavior: inhibition, error detection, and correction. Neuroimage 17:1820-1829.

Gehring WJ, Knight RT (2000) Prefrontal-cingulate interactions in action monitoring. Nat Neurosci 3:516-520.

Gehring WJ, Goss B, Coles MG, Meyer DE, Donchin E (1993) A neural system for error detection and compensation. Psychol Sci 4:385-390.

Gratton G, Coles MG, Donchin E (1983) A new method for off-line removal of ocular artifact. Electroencephalogr Clin Neurophysiol 55:468-484. 
Haber SN (2003) The primate basal ganglia: parallel and integrative networks. J Chem Neuroanat 26:317-330.

Hatanaka N, Tokuno H, Hamada I, Inase M, Ito Y, Imanishi M, Hasegawa N, Akazawa T, Nambu A, Takada M (2003) Thalamocortical and intracortical connections of monkey cingulate motor areas. J Comp Neurol 462:121-138.

Hogan AM, Vargha-Khadem F, Saunders DE, Kirkham FJ, Baldeweg T (2006) Impact of frontal white matter lesions on performance monitoring: ERP evidence for cortical disconnection. Brain 129:2177-2188.

Holroyd CB, Coles MG (2002) The neural basis of human error processing: reinforcement learning, dopamine, and the error-related negativity. Psychol Rev 109:679-709.

Hoshi E, Tremblay L, Féger J, Carras PL, Strick PL (2005) The cerebellum communicates with the basal ganglia. Nat Neurosci 8:1491-1493.

Huynh H, Feld LS (1970) Conditions under which mean square ratios in repeated measurements designs have exact $\mathrm{F}$ distributions. J Am Stat Assoc 65:1582-1589.

Jenkinson M, Bannister P, Brady M, Smith S (2002) Improved optimization for the robust and accurate linear registration and motion correction of brain images. Neuroimage 17:825-841.

Jocham G, Neumann J, Klein TA, Danielmeier C, Ullsperger M (2009) Adaptive coding of action values in the human rostral cingulate zone. J Neurosci 29:7489-7496.

Johannes S, Wieringa BM, Nager W, Müller-Vahl KR, Dengler R, Münte TF (2002) Excessive action monitoring in Tourette syndrome. J Neurol 249:961-966.

Johansen-Berg H, Rushworth MF (2009) Using diffusion imaging to study human connectional anatomy. Annu Rev Neurosci 32:75-94.

Jones DK, Horsfield MA, Simmons A (1999) Optimal strategies for measuring diffusion in anisotropic systems by magnetic resonance imaging. Magn Reson Med 42:515-525.

Jones EG (2007) The thalamus. New York: Cambridge UP.

Kaden E, Knösche TR, Anwander A (2007) Parametric spherical deconvolution: inferring anatomical connectivity using diffusion MR imaging. Neuroimage 37:474-488.

Kennerley SW, Walton ME, Behrens TE, Buckley MJ, Rushworth MF (2006) Optimal decision making and the anterior cingulate cortex. Nat Neurosci 9:940-947.

Kerns JG, Cohen JD, MacDonald AW 3rd, Cho RY, Stenger VA, Carter CS (2004) Anterior cingulate conflict monitoring and adjustments in control. Science 303:1023-1026.

King JA, Korb FM, von Cramon DY, Ullsperger M (2010) Post-error behavioral adjustments are facilitated by activation and suppression of task-relevant and task-irrelevant information processing. J Neurosci 30:12759-12769.

Klein TA, Endrass T, Kathmann N, Neumann J, von Cramon DY, Ullsperger M (2007) Neural correlates of error awareness. Neuroimage 34:1774-1781.

Koch MA, Norris DG, Hund-Georgiadis M (2002) An investigation of functional and anatomical connectivity using magnetic resonance imaging. Neuroimage 16:241-250.

Marceglia S, Servello D, Foffani G, Porta M, Sassi M, Mrakic-Sposta S, Rosa M, Barbieri S, Priori A (2010) Thalamic single-unit and local field potential activity in Tourette syndrome. Mov Disord 25:300-308.

Müller SV, Johannes S, Wieringa B, Weber A, Müller-Vahl K, Matzke M, Kolbe H, Dengler R, Münte TF (2003) Disturbed monitoring and response inhibition in patients with Gilles de la Tourette syndrome and co-morbid obsessive compulsive disorder. Behav Neurol 14:29-37.

Parker GJ, Haroon HA, Wheeler-Kingshott CA (2003) A framework for a streamline-based probabilistic index of connectivity (PICo) using a structural interpretation of MRI diffusion measurements. J Magn Reson Imaging 18:242-254.
Picard N, Strick PL (1996) Motor areas of the medial wall: a review of their location and functional activation. Cereb Cortex 6:342-353.

Rabbitt P (2002) Consciousness is slower than you think. Q J Exp Psychol A 55:1081-1092.

Rabbitt PM (1966) Errors and error correction in choice-response tasks. J Exp Psychol 71:264-272.

Ridderinkhof KR, Ullsperger M, Crone EA, Nieuwenhuis S (2004) The role of the medial frontal cortex in cognitive control. Science 306:443-447.

Rorden C, Karnath HO (2004) Using human brain lesions to infer function: a relic from a past era in the fMRI age? Nat Rev Neurosci 5:813-819.

Rushworth MF, Behrens TE (2008) Choice, uncertainty and value in prefrontal and cingulate cortex. Nat Neurosci 11:389-397.

Servello D, Porta M, Sassi M, Brambilla A, Robertson MM (2008) Deep brain stimulation in 18 patients with severe Gilles de la Tourette syndrome refractory to treatment: the surgery and stimulation. J Neurol Neurosurg Psychiatry 79:136-142.

Servello D, Sassi M, Brambilla A, Porta M, Haq I, Foote KD, Okun MS (2009) De novo and rescue DBS leads for refractory Tourette syndrome patients with severe comorbid OCD: a multiple case report. J Neurol 256:1533-1539.

Shima K, Tanji J (1998) Role for cingulate motor area cells in voluntary movement selection based on reward. Science 282:1335-1338.

Talairach P, Tournoux J (1988) A stereotactic coplanar atlas of the human brain. Stuttgart: Thieme.

Ullsperger M (2006) Performance monitoring in neurological and psychiatric patients. Int J Psychophysiol 59:59-69.

Ullsperger M, von Cramon DY (2006a) How does error correction differ from error signaling? An event-related potential study. Brain Res 1105:102-109.

Ullsperger M, von Cramon DY (2006b) The role of intact frontostriatal circuits in error processing. J Cogn Neurosci 18:651-664.

Ullsperger M, von Cramon DY, Müller NG (2002) Interactions of focal cortical lesions with error processing: evidence from event-related brain potentials. Neuropsychology 16:548-561.

Ullsperger M, Bylsma LM, Botvinick MM (2005) The conflict adaptation effect: it's not just priming. Cogn Affect Behav Neurosci 5:467-472.

Van der Werf YD, Witter MP, Groenewegen HJ (2002) The intralaminar and midline nuclei of the thalamus: anatomical and functional evidence for participation in processes of arousal and awareness. Brain Res Brain Res Rev 39:107-140.

Van Hoesen GW, Morecraft RJ, Vogt BA (1993) Connections of the monkey cingulate cortex. In: Neurobiology of cingulate cortex and limbic thalamus (Vogt BA, Gabriel M, eds), pp 249-284. Boston: Birkhäuser.

Vogt BA (2005) Pain and emotion interactions in subregions of the cingulate gyrus. Nat Rev Neurosci 6:533-544.

Vogt BA, Rosene DL, Pandya DN (1979) Thalamic and cortical afferents differentiate anterior from posterior cingulate cortex in the monkey. Science 204:205-207.

Vogt BA, Sikes RW, Vogt LJ (1993) Anterior cingulate cortex and the medial pain system. In: Neurobiology of cingulate cortex and limbic thalamus: a comprehensive handbook (Vogt BA, Gabriel M, eds), pp 313-344. Boston: Birkhäuser.

Wessel JR, Ullsperger M (2011) Selection of independent components representing event-related brain potentials: a data-driven approach for greater objectivity. Neuroimage 54:2105-2115.

Willemssen R, Müller T, Schwarz M, Hohnsbein J, Falkenstein M (2008) Error processing in patients with Parkinson's disease: the influence of medication state. J Neural Transm 115:461-468.

Yeung N, Botvinick MM, Cohen JD (2004) The neural basis of error detection: conflict monitoring and the error-related negativity. Psychol Rev 111:931-959. 